\title{
Effects of Intermediate Mass Black Holes on Nuclear Star Clusters
}

\section{Citation}

Mastrobuono-Battisti, Alessandra, Hagai B. Perets, and Abraham Loeb. 2014. "EFFECTS OF INTERMEDIATE MASS BLACK HOLES ON NUCLEAR STAR CLUSTERS." The Astrophysical Journal 796 (1): 40. https://doi.org/10.1088/0004-637x/796/1/40.

\section{Permanent link}

http://nrs.harvard.edu/urn-3:HUL.InstRepos:41393189

\section{Terms of Use}

This article was downloaded from Harvard University's DASH repository, and is made available under the terms and conditions applicable to Other Posted Material, as set forth at http:// nrs.harvard.edu/urn-3:HUL.InstRepos:dash.current.terms-of-use\#LAA

\section{Share Your Story}

The Harvard community has made this article openly available.

Please share how this access benefits you. Submit a story.

Accessibility 


\title{
EFFECTS OF INTERMEDIATE MASS BLACK HOLES ON NUCLEAR STAR CLUSTERS
}

\author{
Alessandra Mastrobuono-Battisti ${ }^{1}$, Hagai B. Perets ${ }^{1}$, and Abraham Loeb $^{2}$ \\ ${ }^{1}$ Physics Department, Technion-Israel Institute of Technology, Haifa 32000, Israel \\ ${ }^{2}$ Institute for Theory and Computation, Harvard University, 60 Garden Street, Cambridge, MA 02138, USA \\ Received 2014 March 11; accepted 2014 September 23; published 2014 November 4
}

\begin{abstract}
Nuclear star clusters (NSCs) are dense stellar clusters observed in galactic nuclei, typically hosting a central massive black hole. Here we study the possible formation and evolution of NSCs through the inspiral of multiple star clusters hosting intermediate mass black holes (IMBHs). Using an $\mathrm{N}$-body code, we examine the dynamics of the IMBHs and their effects on the NSC. We find that IMBHs inspiral to the core of the newly formed NSC and segregate there. Although the IMBHs scatter each other and the stars, none of them is ejected from the NSC. The IMBHs are excited to high eccentricities and their radial density profile develops a steep power-law cusp. The stars also develop a power-law cusp (instead of the central core that forms in their absence), but with a shallower slope. The relaxation rate of the NSC is accelerated due to the presence of IMBHs, which act as massive perturbers. This in turn fills the loss cone and boosts the tidal disruption rate of stars both by the MBH and the IMBHs to a value excluded by rate estimates based on current observations. Rate estimates of tidal disruptions can therefore provide a cumulative constraint on the existence of IMBHs in NSCs.
\end{abstract}

Key words: galaxies: bulges - galaxies: kinematics and dynamics - galaxies: nuclei - galaxies: star clusters: general - stars: black holes

Online-only material: color figures

\section{INTRODUCTION}

Nuclear stellar clusters (NSCs) are dense and compact stellar systems observed in galactic nuclei, many of which hosting a massive black hole (MBH) at their center (Böker 2010). Less massive galaxies seem to contain an NSC, but not always an $\mathrm{MBH}$, while the most massive ones show the presence of a central MBH (see, e.g., Greene et al. 2008; Graham \& Spitler 2009; Jiang et al. 2011; Scott \& Graham 2013). Our own Galactic center (GC) hosts both an NSC and a central MBH, Sgr $\mathrm{A}^{*}$ of mass $\mathrm{M}_{\mathrm{BH}} \sim 4 \times 10^{6} M_{\odot}$.

Two main hypotheses have been suggested to explain the origin of NSCs: (1) the in-situ formation, where molecular gas coming from more external regions is channeled to the GC leading to an episodic star formation epoch (Milosavljević 2004); and (2) the cluster-infall/merger scenario, where dense massive stellar clusters decay toward the center of their host galaxy due to dynamical friction, merge and form the NSC. These two scenarios are not mutually exclusive, and both can contribute to the formation of NSCs. Here we focus only the cluster-infall scenario.

The merger scenario was proposed by Tremaine et al. (1975) and later explored in many works in the case of a generic galaxy without a central MBH (see, e.g., Capuzzo-Dolcetta 1993; Agarwal \& Milosavljevic 2011; Capuzzo-Dolcetta \& Miocchi 2008a, 2008b). Other studies explored the case of galaxies hosting an MBH (Antonini 2013), and, in particular, the origin of the NSC in the GC of the Milky Way (Antonini et al. 2012; Gnedin et al. 2014; Perets \& Mastrobuono-Battisti 2014). Relaxed stellar systems around an MBH give rise to a cusp structure (a power-law distribution with a slope of $-7 / 4$, for a single mass population and somewhat shallower slope for most stars in a multi-mass population, Bahcall \& Wolf 1976). However, the relaxation time of the system may be longer than the age of the stars in the NSC, in which case such a cusp may not form. The two-body relaxation time of the observed Galactic NSC, evaluated at the influence radius of the MBH (2-3 pc) is estimated to be $\sim 20-30$ Gyr (Merritt 2010). Current observations of the GC suggest the existence of a flat core in the distribution of the old stellar population of the NSC.

The observed features of the Milky Way NSC have been well reproduced by Antonini et al. (2012) in their $N$-body simulations where they studied 12 consecutive inspirals of massive, compact clusters, which build-up an NSC with a central core of $\sim 0.5 \mathrm{pc}$ and external $\sim r^{-1.8}$ power law outside. Recent studies suggest that this scenario may also lead to age/color and mass segregation in the NSC that can potentially be observed (Perets \& Mastrobuono-Battisti 2014). In analogy with galaxies hosting a central MBH, Silk \& Arons (1975) proposed that massive clusters may host an intermediate mass black hole (IMBH) at their center. The formation of IMBHs in dense clusters has been explored in several studies (Loeb \& Rasio 1994; Madau \& Rees 2001; Ebisuzaki et al. 2001; Bromm $\&$ Loeb 2003). Numerical simulations of the formation and evolution of young dense clusters show that runaway collisions between stars can produce a massive $\operatorname{star}\left(\sim 10^{2}-10^{4} M_{\odot}\right)$ that sinks to the center of the cluster due to dynamical friction (Gürkan et al. 2004; Portegies Zwart et al. 2004; Freitag et al. 2006; Portegies Zwart et al. 2006; Fujii et al. 2009), and later collapses to form an IMBH with a mass of $\sim 10^{2}-10^{4} M_{\odot}$. Given the possible existence of such IMBHs in massive clusters, the cluster-infall scenario may be significantly affected by such objects. Here we extend the cluster-infall scenario to include this possibility, and study the effects of IMBHs on the NSC formation and evolution.

We use $N$-body simulations similar to those described in Antonini et al. (2012), but populate the infalling clusters (ICs) with IMBHs. The outline of the paper is as follows. We begin with a description of the initial conditions and our methods in Section 2. We then present our results (Section 3), discuss their implications (Section 4), and summarize (Section 5). 


\section{INITIAL CONDITIONS AND METHODS}

In our simulations we used the same models and methods described in Paper I (Antonini et al. 2012), where a detailed description of the initial conditions of initial ICs, as well as the galaxy model for the background stellar population, can be found. In brief, we followed the decay and the merging of 12 massive dense clusters inside the Galactic bulge by means of fully self-consistent $N$-body simulations. The bulge of the simulated galaxy has been modeled based on recent observations (Launhardt et al. 2002). A central MBH as massive as the SgrA* is placed at the center of the Galaxy. A truncated power-law model has been used for this component:

$$
\rho_{g}(r)=\tilde{\rho}\left(\frac{r}{\tilde{r}}\right)^{-\gamma} \operatorname{sech}\left(\frac{r}{r_{\text {cut }}}\right) .
$$

where $\tilde{\rho}=400 M_{\odot} \mathrm{pc}^{-3}$ and $r_{\text {cut }}=22 \mathrm{pc}$, thus the galactic mass is $9.1 \times 10^{7} M_{\odot}$. Finally, as in Paper I, $\gamma$ is set to $1 / 2$.

The Monte Carlo method described in Szell et al. (2005) has been used to sample the particles, whose mass is fixed to be $400 M_{\odot}$, as to have $N_{g}=227523$ super stars.

The IC modeling follows Paper I with the addition of a central IMBH of $10^{4} M_{\odot}$. In particular, we used a highly compact and initially massive tidally truncated King model (see Paper I for details on the truncation method). The central velocity dispersion of the model is $\sigma_{0}=35 \mathrm{~km} \mathrm{~s}^{-1}$, the core radius $r_{c}=0.5 \mathrm{pc}$, and $W_{0}=8$. After the truncation the cluster mass is $\sim 1.1 \times 10^{6} M_{\odot}$. We sampled the model using $N_{I C}=5715$, $200 M_{\odot}$ particles.

The ICs are compact and their cumulative mass $(\sim 1.5 \times$ $10^{7} M_{\odot}$ ) is comparable to the mass of the Galactic NSC (Genzel et al. 2010). Massive ICs decline to the galactic nucleus in a time significantly shorter than a Hubble time (Antonini et al. 2012; Capuzzo-Dolcetta 1993). The clusters are given time to relax in isolation before they are put into the cluster-infall simulation. The relaxation timescale is relatively short and is meant to assure the stability of the system.

We let 12 ICs inspiral toward the GC in a consecutive order. A new IC is sent only after the previous one has merged at the center of the Galaxy and has reached a quasi-stationary state. We follow the ICs after they have already reached the central region of the galaxy. Each IC is initially placed on a circular orbit with a radius of $20 \mathrm{pc}$ from the $\mathrm{MBH}$, as described in Gualandris \& Merritt (2009) and in Paper I.

We ran our simulations with the $\phi G R A P E$ code (Harfst et al. 2007), a direct-summation code optimized for computer clusters accelerated by GRAPE boards (Makino \& Taiji 1998). The time integrator for this code is a fourth-order Hermite with a predictor-corrector scheme and hierarchical time stepping. $\phi G R A P E$ has been recently adapted to run on graphic processing units (GPUs) by means of Sapporo, a CUDA library that emulates double-precision force calculations on singleprecision hardwares (Gaburov et al. 2009). The accuracy and performance of the code are set by the time-step parameter $\eta$ (the time step is proportional to the square root of $\eta$ ) and the smoothing length $\epsilon$. In our simulations we chose $\eta=0.01$ and $\epsilon=0.01 \mathrm{pc}=2 \times 10^{-2} r_{c}$. With this choice we have a relative energy conservation of less than $10^{-4}$ until the tenth merging event, and $\sim 10^{-3}$ for the remaining part of the simulation. This decay in energy conservation is probably due to the interaction between stars and IMBHs and between the IMBHs. The simulations have been run on the GPU partition of the Tamnun cluster at the Technion. This partition consists of four nodes, each equipped with one NVidia Tesla M2090. Since $\phi G R A P E$ can run on multiple GPUs only if they are installed on the same node, we used one GPU for each run. Each simulation needed at least four months to be completed. Due to computational limitations, the ICs were represented by a smaller number of particles than realistic systems, each with a higher particle mass as to represent the total cluster mass (as discussed above). The simulation time was then rescaled to real time using the procedure described in detail by Mastrobuono-Battisti \& Perets (2013) and Perets \& Mastrobuono-Battisti (2014).

We followed the inspiral of the $12 \mathrm{ICs}$ toward the GC until they were tidally destroyed by the $\mathrm{MBH}$ and showed a quasi-stationary state. In order to support the reliability and reproducibility of our results we have run two additional simulations using, as initial conditions, different samplings of the same initial physical conditions. Here we show only results from the first run, but these were verified with the additional runs, both showing very similar results. In Figure 3, the density profile for the final NSC is shown for all three simulations. As it is apparent from the plot, the curves do not show any significant difference supporting our conclusions. Figure 2 shows the Lagrangian radii of the first decaying cluster, evaluated in respect to the center of density (Casertano \& Hut 1985) of the cluster itself. After the cluster is destroyed, we waited for the Lagrangian radii to be almost constant before sending the following cluster. After the end of all the infalls, we followed the relaxation process of the final system. This relaxation is driven by two-body interactions between stars, stars and IMBHs, and among the IMBHs.

\section{RESULTS}

\subsection{The Density Profile and Kinematic Properties}

To follow the formation of the central NSC, we plot the density profile after each merging. Figure 1 shows the density profile of the stellar component of the system (galaxy+stars in the clusters, left panel) and of the IMBHs (right panel) after 3, 6, 9, and 12 merging events. While the background bulge does not change its profile, the central region evolves toward an NSC structure. The presence of the IMBHs significantly changes the properties and evolution of the NSC. In particular, in the simulations run without the central IMBH (Antonini et al. 2012), the ICs are disrupted at $\sim 2$ pc (see the red line in Figure 2), and their stars develop a central flat core (see the red solid line in the left panel of Figure 1). In comparison, following the disruption of the IMBH-hosting ICs, the IMBHs keep inspiraling to the center and lead to the fast relaxation of the NSC stellar population, giving rise to a central cusp, with a somewhat shallower slope than a Bahcall \& Wolf (1976) profile, likely due to the strong mass segregation induced by the IMBHs (see Section 4.2). As can be seen in the left panel of Figure 1, a nearly flat core with radius $\sim 1 \mathrm{pc}$ still exists, but by the sixth IC infall a central cusp has already formed, both in the stellar and IMBHs components (right panel of Figure 1).

At the end of the last infall the inner slope of the radial density profile for the whole system (Galaxy+NSC) is $\sim-2.1$, i.e., significantly different than the density profile of the IMBHfree cluster-infall scenario (Antonini et al. 2012; Perets \& Mastrobuono-Battisti 2014), where the NSC shows a central core. Although the core size decreases with each infall, it still exists even after the long-term evolution and further relaxation of the system. Indeed, the IMBHs act as massive perturbers, and thereby significantly decrease the relaxation time of the 

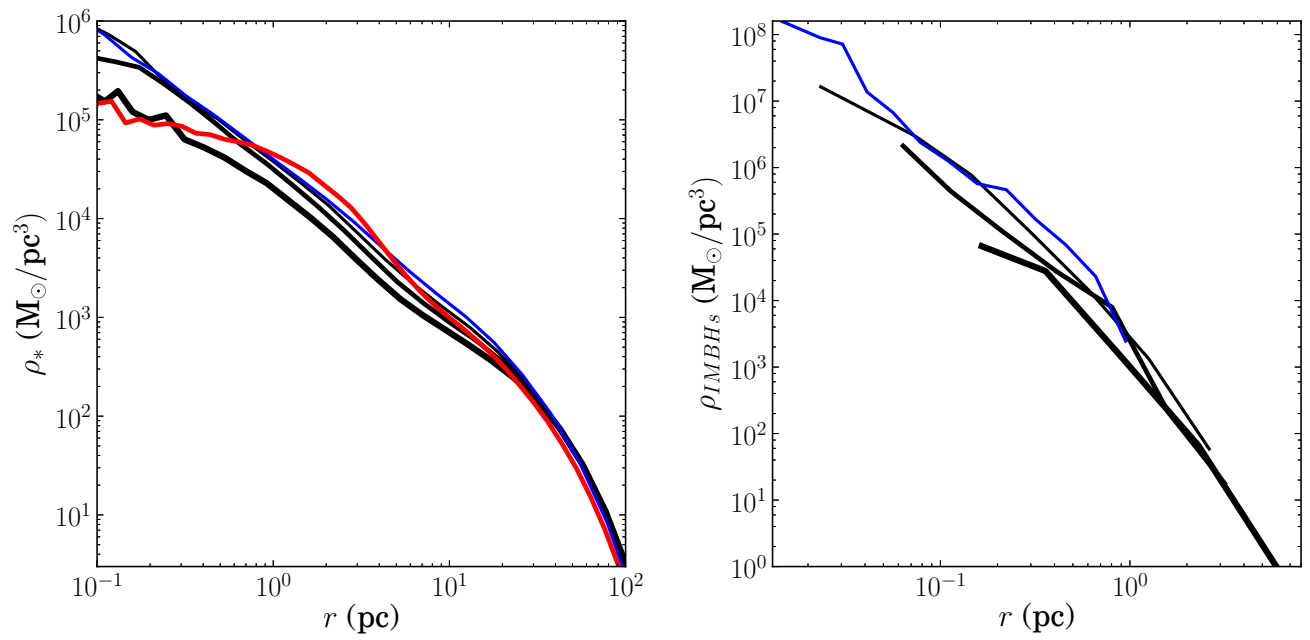

Figure 1. Left panel: the spatial density profile of the stellar component of the growing NSC after 3, 6, 9, and 12 decays. The thickness of lines decreases with time and the blue solid line is the density profile after 12 merging events. The red solid line represents the density profile of the NSC obtained after 12 merging events in Paper I. With IMBHs the NSC develops a density cusp whereas without IMBHs the NSC has a central core. Right panel: same for the density profile of IMBHs.

(A color version of this figure is available in the online journal.)

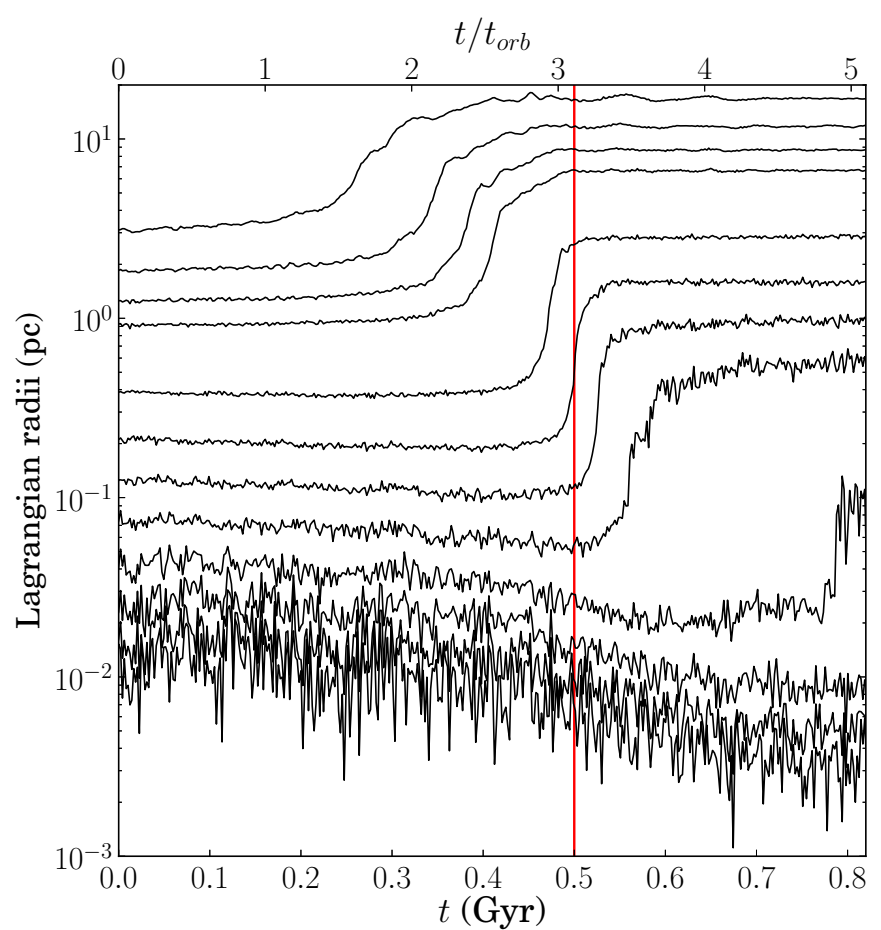

Figure 2. Lagrangian radii of stars from the first decaying cluster in respect to the center of density of the cluster itself. The percentage of mass enclosed within each of the plotted radii are: $0.025 \%, 0.06 \%, 0.16 \%, 0.4 \%, 1 \%, 2.5 \%$, $6.3 \%, 16 \%, 40 \%, 50 \%, 63 \%$, and $79 \%$. The time given on the bottom $x$ axis is rescaled with the mass of the particles and softening length used. On the top $x$ axis, we show the time in terms of the period of the circular orbit at $20 \mathrm{pc}$, i.e., the starting distance of the ICs. The red vertical line shows when the center of density of the system is at $2 \mathrm{pc}$ from the central MBH. This time roughly corresponds to the disruption time of the cluster.

(A color version of this figure is available in the online journal.)

host system (Perets et al. 2007). At the end of the simulation, i.e., after $12 \mathrm{Gyr}(\sim 400$ internal crossing times of the ICs), both the stellar cusp and steep radial distribution of the IMBHs are fully developed (see Figure 3 and Section 4.2 for more details).

The kinematic properties of the NSC in the cluster infall with the IMBHs simulations are similar to those found in Paper I. At

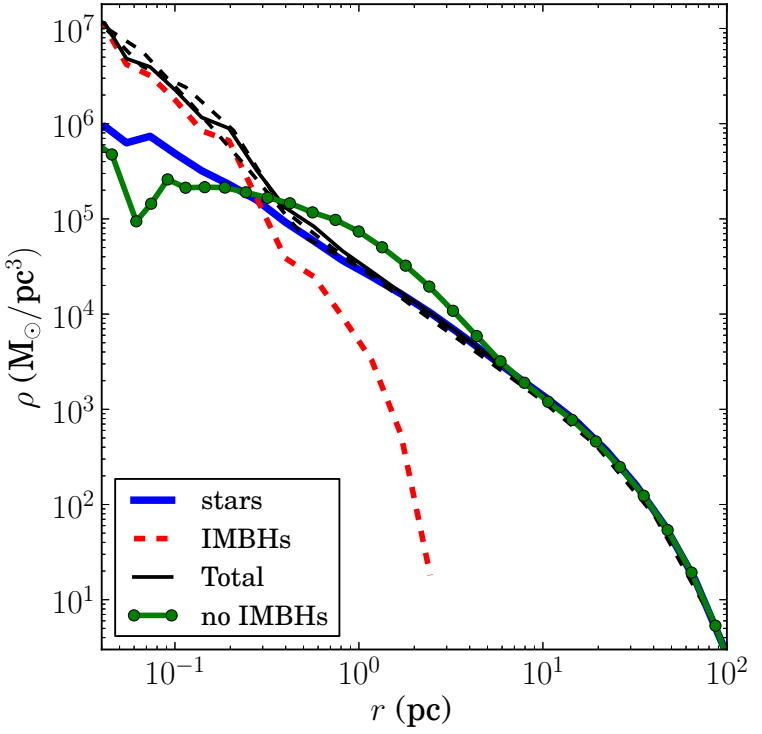

Figure 3. Density profiles of the NSC at the end of the simulation (black solid line). We run two additional simulations to check our results. The density profiles of the NSCs obtained in these cases are shown by the black dashed lines. The blue solid line represents the spatial profile of the stellar component, while the red dashed line is the final density profile of the $12 \mathrm{IMBHs}$. The green solid line with bullets shows the density profile of the NSC without IMBHs (from Paper I). The IMBHs introduce a central cusp due to enhanced relaxation.

(A color version of this figure is available in the online journal.)

the end of the last merger, the NSC is tangentially anisotropic between 0.3 and $30 \mathrm{pc}$, and it is almost isotropic outside this radius. Since the cluster evolves toward isotropy, the tangential anisotropy decreases in the subsequent 2 Gyr of evolution. This is also seen in the axial ratios and in the triaxiality parameter: the cluster remains oblate even after the mergers evolution, within $30 \mathrm{pc}$. As found earlier, outside this radius the system retains its initial spherical configuration.

\subsection{Dynamical Evolution of the IMBHs}

As shown in the previous section, the presence of IMBHs significantly affects the inner structure of the NSC. Figure 4 shows the galactocentric distance of the last IMBH to decrease 


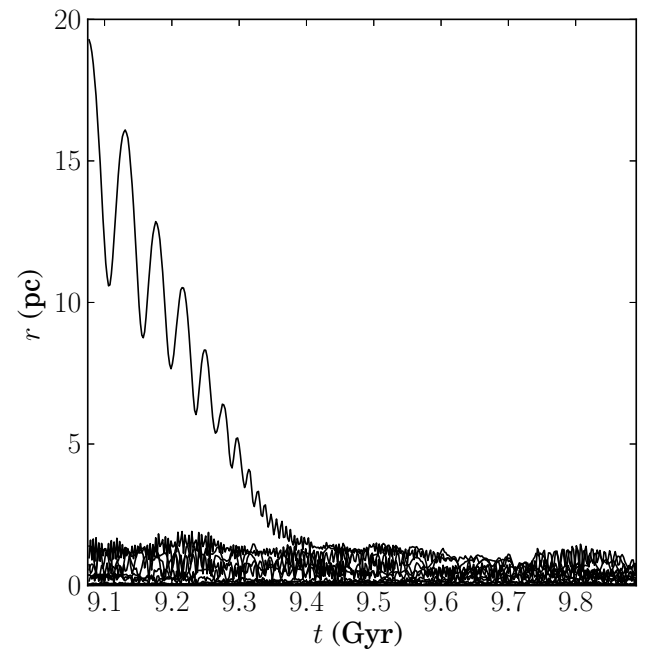

Figure 4. Upper line shows the temporal decay of the orbital radius of the last IMBH to fall in. The radius vs. time of the other $11 \mathrm{IMBHs}$ is also shown.

as a function of time. The IMBH initially decays with its host IC, until the cluster is tidally disrupted. The IMBH then continues to inspiral by itself due to dynamical friction induced by the NSC stars. The other curves in Figure 4 represent the distance from the GC of the previous IMBHs that already settled to the center. Though the IMBHs interact and scatter each other, they are never ejected from the system and are contained within the central 2 pc during the post-merger evolution.

Figure 5 shows the eccentricity distribution of the IMBHs at the end of the simulation. The IMBHs can reach high eccentricities, with values close to unity due to mutual scatterings.

\section{DISCUSSION}

\subsection{Fast Relaxation and a High Rate of Tidal Disruption Events}

The presence of IMBHs in ICs significantly affects the formation and evolution of NSCs. The inspiral of the IMBHs to the nucleus of the NSC both provides additional stars to the central region of the NSC, and leads to its faster relaxation. Acceleration of relaxation processes can enhance the rate of scattering stars into highly eccentric orbits and the refilling of the loss cone for tidal disruption events (TDEs) of stars (see also Madigan \& Levin 2012). Following Perets et al. (2007), we evaluated the local empty and full loss cone rates, using stellar number density profiles based on the mass density profiles from our simulations (assuming the stellar mass divided among Solar mass stars)

$$
\begin{aligned}
\frac{d \Gamma_{e}}{d \log r} \sim \frac{N_{\star}(<r)}{\ln \left(J_{c} / J_{l c}\right) t_{r}(r)}, \\
\frac{d \Gamma_{f}}{d \log r} \sim \frac{J_{l c}^{2}}{J_{c}^{2}} \frac{N_{\star}(<r)}{P(r)},
\end{aligned}
$$

where $N_{\star}(<r)$ is the number of stars enclosed within $r, t_{r}(r)$ is the relaxation time at $r, J_{l c}$ and $J_{c}$ are, respectively, the loss cone angular momentum and the angular momentum of the circular orbit of radius $r$ and, finally, $P(r)$ is the Keplerian period at $r$.

The total loss cone refilling rate is then

$$
\Gamma_{\text {tot }}=\int \frac{d \Gamma}{d r} d r=\int \min \left[\frac{d \Gamma_{f}}{d r}, \frac{d \Gamma_{e}}{d r}\right] d r .
$$

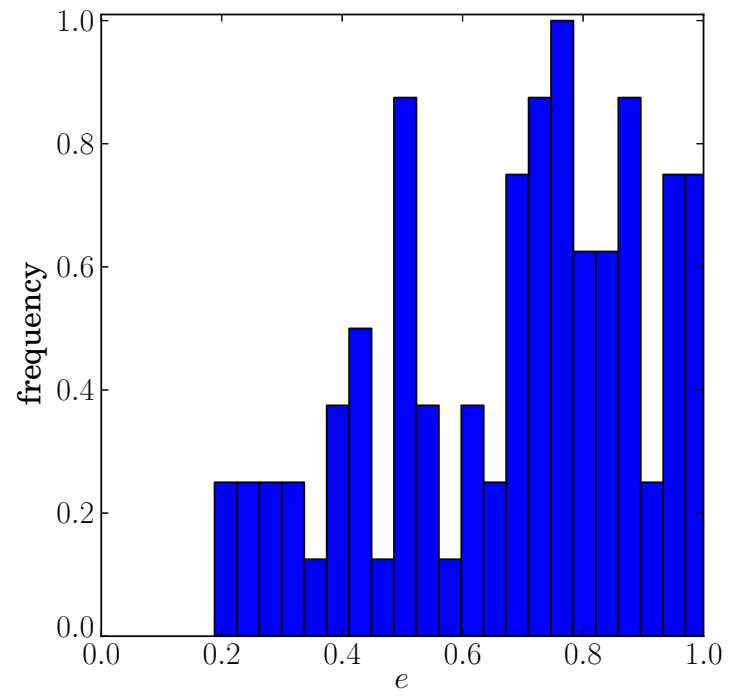

Figure 5. Eccentricity distribution of the IMBHs. Multiple snapshots close in time have been used to increase the data resolution.

(A color version of this figure is available in the online journal.)

The presence of IMBHs in the NSC decreases the relaxation time by a factor of $\propto N_{I} M_{I}^{2} /\left(N_{\star} M_{\star}^{2}\right)$, where $N_{I}$ and $M_{I}$ are the number and the mass of the IMBHs, while $N_{\star}$ and $M_{\star}$ are the corresponding number and mass of the stars. The relaxation time is, therefore, shortened by a few orders of magnitude, allowing for the rapid relaxation of the system. This also significantly affects the loss cone refilling rate for the tidal disruption of stars by the MBH. With the IMBHs, the system is in the full loss cone regime throughout all of its radial extent, while, without them, the loss cone is empty inside the radius of influence of the $\mathrm{MBH}$. The integrated TDE rate with the IMBHs is $10^{-3} \mathrm{yr}^{-1}$, while otherwise, accounting only for stars, we find a rate of $1.6 \times 10^{-5} \mathrm{yr}^{-1}$, comparable to the typical rate estimated for NSCs similar to that of the Milky Way (Merritt 2013). The inspiral of IMBHs can, therefore, increase the TDE rate by two orders of magnitude. It is interesting to notice that a similar result has been obtained by Chen \& Liu (2013) using purely analytical calculations.

The inferred rate of TDEs from observations is of the order of $10^{-5}-10^{-4} \mathrm{yr}^{-1}$ (see Komossa 2012; Khabibullin \& Sazonov 2014, and references therein), much lower than expected for multiple-IMBHs hosting NSCs such as those seen in our simulations. We, therefore, conclude that typical NSCs should not contain IMBHs. If cluster infall is one of the main channels for NSC formation, this also implies that the majority of massive clusters may not host IMBHs. We do caution that the observational rate estimates are still inaccurate; better data will be available from future large surveys.

The presence of an IMBH could also induce an enhancement in the number of stars plunging to the $\mathrm{MBH}$ through secular Kozai evolution, if the IMBH reaches close enough to the $\mathrm{MBH}$ to provide a significant perturbation, not quenched by the mass precession from the background stars (see, e.g., Chen et al. 2011; Stone \& Loeb 2012; Chen \& Liu 2013; Liu \& Chen 2013). This could happen only during the last stages of inspiral. In our case, the IMBHs do not attain such a configuration, thus the massive perturbers' fast relaxation dominates the enhanced rate of TDEs by the $\mathrm{MBH}$.

Besides their contribution to the TDE rate by the $\mathrm{MBH}$, IMBHs can, by themselves, directly disrupt stars. The rate 
of TDEs by the IMBHs can be readily estimated by the rate of random close encounters of stars with the IMBHs.

$$
\Gamma_{I}=4 \pi \int r^{2} n_{*}(r) \sigma(r) v_{*}(r) n_{I}(r) d r,
$$

where $n_{*}(r)$ is the stellar number density, $\sigma(r)$ is the cross section for the IMBHs including gravitational focusing, evaluated using the tidal disruption radius of the IMBHs, $v_{*}(r)$ is the stellar velocity as a function of the radius and $n_{I}(r)$ is the IMBHs number density. For our system, at the end of the last merging, Equation (5) gives $\Gamma_{I} \sim 10^{-3} \mathrm{yr}^{-1}$, a total rate of TDEs by all IMBHs, which is comparable with the one due to the MBH alone. Thus, in this scenario we expect both TDEs by the MBH and by the IMBHs. Interestingly, these two sources will produce different signatures (see Lodato 2012, and references therein). The IMBH contribution can be long lived, since they apparently survive in the central $2 \mathrm{pc}$ and they do not inspiral to the $\mathrm{MBH}$, nor are they ejected from nucleus.

\subsection{Strong Mass Segregation and the Cusp Structure and Evolution}

As discussed in Section 3, the central density cusp is already established after the sixth infall. The density grows with the number of infalls while the slope of the cusp remains almost constant. Following the final merger of the ICs, the system evolves due to two-body relaxation dominated by the IMBHs. The stellar component slightly changes its slope, while the IMBHs distribution maintains its initial slope.

Since the number density of IMBHs is much lower than that of stars, we expect the system to be in the strong mass segregation regime (Alexander \& Hopman 2009). Indeed, the two populations settle to different density profiles with different slopes. After rescaling the simulation time with the relaxation time of the system, we find that it extends to $\sim 12 \mathrm{Gyr}$. At the end of the simulation, the system distribution of the IMBHs (high-mass particles) is well-fitted by a power law,

$$
\rho_{H}(r)=6.20 \times 10^{3} r^{-2.32} M_{\odot} \mathrm{pc}^{-3},
$$

(see Figure 3). The slope of the IMBH cusp is, therefore, within the range expected in the strong mass segregation case for the higher mass particles $\left(2 \lesssim \alpha_{H} \lesssim 11 / 4\right.$; see Alexander $\&$ Hopman 2009). Note that the IMBH density profile, shown in both the right panel of Figures 1 and 3, includes data on a small number of objects, and may therefore be sensitive to significant statistical noise, and the exact power-law profile calculated should be taken with caution. Nevertheless, all three simulations we have run show consistent results, and very similar powerlaw profiles clearly indicating a cusp profile for the IMBHs compared with the core profile of the stellar population. The stellar component can be fitted by a shallower radial profile,

$$
\rho_{L}(r)=1.4 \times 10^{5} r^{-1.26},
$$

whose power-law index is somewhat shallower than expected $\left(3 / 2 \lesssim \alpha_{L} \lesssim 7 / 4\right)$.

These results qualitatively differ from those obtained for IMBH-free ICs case for which we found the stellar population of the NSC to have an external power-law distribution with a slope of $\sim-1.8$ outside an inner core that shrinks with time but still exists out to $0.5 \mathrm{pc}$ at the end of the simulation. With IMBHs, the NSC develops the central power-law cusp early in its evolution.

\subsection{Mass Segregation, Compact Objects, and Gravitational Wave Sources}

The inspiral of compact objects onto MBHs is a potential source of gravitational waves (GWs). For MBHs with masses comparable to $\mathrm{Sgr} \mathrm{A}^{*}$, the GW sources associated with extreme mass ratio inspirals (EMRIs) might be detectable by future GW observatories, such as the proposed Laser Interferometer Space Antenna (eLISA ${ }^{3}$; Amaro-Seoane et al. 2007; Amaro-Seoane \& Preto 2011). Stars are tidally destroyed before they enter the Schwarzschild radius of the $\mathrm{MBH}$, while compact objects such as stellar black holes, neutron stars and white dwarfs can survive to eventually produce EMRI GW events. IMBHs may also be affected by GW emission during a close approach to $\mathrm{MBH}$, and their inspiral would have a much larger mass ratio, i.e., producing "intermediate mass ratio inspiral" (IMRI) events. Such inspirals are among the most interesting GW sources that are expected to be detected by eLISA.

The decay time due to GWs emission from an initial semimajor axis $a$ is given by

$$
t_{G W}=\frac{5}{256} \frac{c^{5}}{G^{3}} \frac{a^{4}}{\mu M_{12}^{2}},
$$

where $\mu$ is the reduced mass of the system, while $M_{12}$ is the mass of the binary (Peters 1964; Perets \& Alexander 2008). Figure 5 shows that the IMBHs can reach very high eccentricities, and their evaluated GW inspiral time can be much shorter than a Hubble time, but typically much longer than the time between strong scattering by other IMBHs. Such scatterings can increase the IMBH separation from the MBH. Our simulations do not account for the GW emission, and therefore it is not clear whether any of the IMBHs is likely to produce IMRI GW sources. To check this effect, we evaluated the ratio between the analytically estimated change in energy due to GW emission, and the energy variation due to scattering. This ratio, during the relaxation of the system, is always $\ll 1$, i.e., relaxation dominates the IMBH dynamical evolution, and the IMBHs are unlikely to inspiral and become GW sources. Future simulations with GW emission effects could shed more light on this possibility.

As already found by Portegies Zwart et al. (2004), the stars that are most bound to an infalling IMBH (which are typically the most massive, due to mass segregation in the IC), can still accompany the IMBH as it inspirals to the NSC center, even after the disruption of the cluster bulk. Each particle in our ICs is $200 M_{\odot}$; we find that the total mass in bound stars (typically 10 stars) brought in with each IMBH is $\sim 2000 M_{\odot}$. Later on, as the IMBHs reach the central region, even these stars are typically stripped and left behind. IMBHs can therefore induce a more extreme mass segregation than suggested by the IMBH-free scenario (Perets \& Mastrobuono-Battisti 2014; Antonini 2014), and help refill the central NSC core, and assist the development of the central stellar cusp. Since IMBH-hosting ICs are expected to be mass segregated (Bahcall \& Wolf 1977), stellar black holes are likely to reside closest to the IMBH. These stellar black holes can, therefore, be brought by the IMBHs to the NSC center. Such compact objects in the closest regions to the MBH could, therefore, potentially contribute to the EMRI GW rate (Hopman \& Alexander 2006; O’Leary et al. 2009).

\footnotetext{
3 http://www.elisa.org/
} 


\section{SUMMARY AND CONCLUSIONS}

In this work, we explored the formation and evolution of NSCs through the merging of ICs containing IMBHs. The presence of IMBHs substantially modifies the structure of the NSC. Our main results are as follows.

1. Following the infall and disruption of an inspiraling cluster, its IMBH continues to spiral in, accompanied by a small group of stars or compact objects, which are later stripped from the IMBH close to the central MBH. The IMBHs later scatter each other and the NSC stars, and can develop high eccentricities.

2. In contrast to the IMBH-free case, the NSC develops a cusp already in its early evolutionary stages.

3. The system is strongly mass-segregated: IMBHs and stars evolve separately and settle on two different quasistationary profiles characterized by a central cusp with different slopes.

4. The NSC is tangentially anisotropic and oblate, very similar to simulated NSCs without IMBHs.

5. The IMBHs decrease the relaxation time of the NSC by a factor of a few hundred, leading to the fast refilling of the loss cone for tidal disruption of stars by the central $\mathrm{MBH}$. The system is in the full loss cone regime and the tidal disruption rates are two orders of magnitude larger than in an NSC without IMBHs. In addition, the rate of TDEs by the IMBHs themselves is comparable to that of the central $\mathrm{MBH}$.

6. The large rate of TDEs expected from IMBHs-hosting NSCs compared to the much lower TDE rate estimates from observations, suggests that typical NSCs likely do not contain IMBHs. If cluster-infall is one of the main channels for NSC formation, this also implies that the majority of massive clusters do not host IMBHs. We do caution, however, that the accuracy and reliability of current TDE rate estimates is still limited, and better estimates would be available from future surveys.

This research was supported by the I-CORE Program of the Planning and Budgeting Committee and The Israel Science Foundation grant $1829 / 12$, and in part by the Technion by a fellowship from the Lady Davis Foundation. The work was also supported by NSF grant AST-1312034.

\section{REFERENCES}

Agarwal, M., \& Milosavljevic, M. 2011, ApJ, 729, 35

Alexander, T., \& Hopman, C. 2009, ApJ, 697, 1861

Amaro-Seoane, P., Gair, J. R., Freitag, M., et al. 2007, CQGra, 24, R113

Amaro-Seoane, P., \& Preto, M. 2011, CQGra, 28, 094017
Antonini, F. 2013, ApJ, 763, 62

Antonini, F. 2014, ApJ, 794, 106

Antonini, F., Capuzzo-Dolcetta, R., Mastrobuono-Battisti, A., \& Merritt, D. 2012, ApJ, 750, 111 (Paper I)

Bahcall, J. N., \& Wolf, R. A. 1976, ApJ, 209, 214

Bahcall, J. N., \& Wolf, R. A. 1977, ApJ, 216, 883

Böker, T. 2010, in Astrophysics and Space Science Proc., The Impact of HST on European Astronomy, ed. W. Burton, L. L. Christensen, \& F. D. Macchetto (Berlin: Springer), 99

Bromm, V., \& Loeb, A. 2003, ApJ, 596, 34

Capuzzo-Dolcetta, R. 1993, ApJ, 415, 616

Capuzzo-Dolcetta, R., \& Miocchi, P. 2008a, MNRAS, 388, L69

Capuzzo-Dolcetta, R., \& Miocchi, P. 2008b, ApJ, 681, 1136

Casertano, S., \& Hut, P. 1985, ApJ, 298, 80

Chen, X., \& Liu, F. L. 2013, ApJ, 762, 95

Chen, X., Sesana, A., Madau, P., \& Liu, F. K. 2011, ApJL, 729, L13

Ebisuzaki, T., Makino, J., Tsuru, T. G., et al. 2001, ApJL, 562, L19

Freitag, M., Gürkan, M. A., \& Rasio, F. A. 2006, MNRAS, 368, 141

Fujii, M., Iwasawa, M., Funato, Y., \& Makino, J. 2009, ApJ, 695, 1421

Gaburov, E., Harfst, S., \& Portegies Zwart, S. 2009, NewA, 14, 630

Genzel, R., Eisenhauer, F., \& Gillessen, S. 2010, RvMP, 82, 3121

Gnedin, O. Y., Ostriker, J. P., \& Tremaine, S. 2014, ApJ, 785, 71

Graham, A. W., \& Spitler, L. R. 2009, MNRAS, 397, 2148

Greene, J. E., Ho, L. C., \& Barth, A. J. 2008, ApJ, 688, 159

Gualandris, A., \& Merritt, D. 2009, ApJ, 705, 361

Gürkan, M. A., Freitag, M., \& Rasio, F. A. 2004, ApJ, 604, 632

Harfst, S., Gualandris, A., Merritt, D., et al. 2007, NewA, 12, 357

Hopman, C., \& Alexander, T. 2006, ApJL, 645, L133

Jiang, Y., Greene, J. E., \& Ho, L. C. 2011, ApJL, 737, L45

Khabibullin, I., \& Sazonov, S. 2014, MNRAS, 444, 1041

Komossa, S. 2012, in EPJ Web of Conferences, Vol. 39, Tidal Disruption Events and AGN Outbursts, ed. R. Saxton \& S. Komossa (Madrid, Spain: EPJ Web of Conferences), 02001

Launhardt, R., Zylka, R., \& Mezger, P. G. 2002, A\&A, 384, 112

Liu, F. K., \& Chen, X. 2013, ApJ, 767, 18

Lodato, G. 2012, in EPJ Web of Conferences, Vol. 39, Tidal Disruption Events and AGN Outbursts, ed. R. Saxton \& S. Komossa (Madrid, Spain: EPJ Web of Conferences), 01001

Loeb, A., \& Rasio, F. A. 1994, ApJ, 432, 52

Madau, P., \& Rees, M. J. 2001, ApJL, 551, L27

Madigan, A. M., \& Levin, Y. 2012, ApJ, 754, 42

Makino, J., \& Taiji, M. (ed.) 1998, Scientific Simulations with Special-purpose Computers. The GRAPE Systems (New York: Wiley-VCH), 248

Mastrobuono-Battisti, A., \& Perets, H. B. 2013, ApJ, 779, 85

Merritt, D. 2010, ApJ, 718, 739

Merritt, D. 2013, CQGra, 30, 24

Milosavljević, M. 2004, ApJL, 605, L13

O’Leary, R., Kocsis, B., \& Loeb, A. 2009, MNRAS, 395, 2127

Perets, H. B., \& Alexander, T. 2008, ApJ, 677, 146

Perets, H. B., Hopman, C., \& Alexander, T. 2007, ApJ, 656, 709

Perets, H. B., \& Mastrobuono-Battisti, A. 2014, ApJL, 784, L44

Peters, P. C. 1964, PhRv, 136, 1224

Portegies Zwart, S. F., Baumgardt, H., Hut, P., Makino, J., \& McMillan, S. L. W. 2004, Natur, 428, 724

Portegies Zwart, S. F., Baumgardt, H., McMillan, S. L. W., et al. 2006, ApJ, 641,319

Scott, N., \& Graham, A. W. 2013, ApJ, 763, 76

Silk, J., \& Arons, J. 1975, ApJL, 200, L131

Stone, N., \& Loeb, A. 2012, MNRAS, 422, 1933

Szell, A., Merritt, D., \& Kevrekidis, I. G. 2005, PhRvL, 95, 081102

Tremaine, S. D., Ostriker, J. P., \& Spitzer, L. 1975, ApJ, 196, 407 